\title{
THE ENERGY CHANGE OF THE COMPLETE MULTIPARTITE GRAPH*
}

\author{
HAI-YING SHAN ${ }^{\dagger}$, CHANG-XIANG HE ${ }^{\ddagger}$, AND ZHEN-SHENG YU ${ }^{\ddagger}$
}

\begin{abstract}
The energy of a graph is defined as the sum of the absolute values of all eigenvalues of the graph. Akbari et al. [S. Akbari, E. Ghorbani, and M. Oboudi. Edge addition, singular values, and energy of graphs and matrices. Linear Algebra Appl., 430:2192-2199, 2009.] proved that for a complete multipartite graph $K_{t_{1}, \ldots, t_{k}}$, if $t_{i} \geq 2(i=1, \ldots, k)$, then deleting any edge will increase the energy. A natural question is how the energy changes when $\min \left\{t_{1}, \ldots, t_{k}\right\}=1$. In this paper, a new method to study the energy of graph is explored. As an application of this new method, the above natural question is answered and it is completely determined how the energy of a complete multipartite graph changes when one edge is removed.
\end{abstract}

Key words. Graph, Complete multipartite graph, Energy.

AMS subject classifications. 15A15, 15F10.

1. Introduction. Let $G=(V, E)$ be a simple connected graph with vertex set $V=\left\{v_{1}, v_{2}, \ldots, v_{n}\right\}$ and edge set $E$. The adjacency matrix of $G, A(G)=\left(a_{i j}\right)$, is an $n \times n$ matrix, where $a_{i j}=1$ if $v_{i}$ and $v_{j}$ are adjacent and $a_{i j}=0$, otherwise. Thus, $A(G)$ is a real symmetric matrix with zeros on the diagonal, and all eigenvalues of $A(G)$ are real. The characteristic polynomial $\operatorname{det}(x I-A(G))$ of the adjacency matrix $A(G)$ of a graph $G$ is also called the characteristic polynomial of $G$, denoted by $\Phi(G, x)$ or $\Phi(G)$. The eigenvalues of graph $G$ are the eigenvalues of $A(G)$, written as $\lambda_{1}(G) \geq \lambda_{2}(G) \geq \cdots \geq \lambda_{n}(G)$. The energy of $G$, denoted by $\mathcal{E}(G)$, is defined $[4,5]$ as $\mathcal{E}(G)=\sum_{i=1}^{n}\left|\lambda_{i}(G)\right|$.

For the polynomial $f(x)$, if all the roots of $f(x)=0$ are real, we also define the energy of $f(x)$ as the sum of the absolute values of its roots, denoted by $\mathcal{E}(f)$.

We denote a complete multipartite graph with $k \geq 2$ parts by $K_{t_{1}, \ldots, t_{k}}$, where $t_{i}(i=1, \ldots, k)$ is the number of vertices in the $i$-th part of the graph, and we write the $i$-th part as $t_{i}$-part.

One area in the study of graph energy, called graph energy change is to understand how graph energy changes when a subgraph is deleted. It becomes especially interesting when the subgraph is just an edge. As we know the energy of a graph may increase, decrease, or remain the same when an edge is deleted. For more details see [3] and the references therein.

Akbari, Ghorbani and Oboudi [1] (see Theorem 4) proved that for any complete multipartite graph $K_{t_{1}, \ldots, t_{k}}$ with $k \geq 2, t_{i} \geq 2$, then $\mathcal{E}\left(K_{t_{1}, \ldots, t_{k}}-e\right)>\mathcal{E}\left(K_{t_{1}, \ldots, t_{k}}\right)$ for any edge $e$. Then a natural question is how the energy changes when $\min \left\{t_{1}, \ldots, t_{k}\right\}=1$. In this paper, we will answer this question and completely determine how the energy of a complete multipartite graph changes when one edge is deleted. Our main result is

${ }^{*}$ Received by the editors on November 17, 2018. Accepted for publication on August 4, 2019. Handling Editor: Bryan L. Shader. Corresponding Author: Chang-Xiang He.

${ }^{\dagger}$ School of Mathematical Sciences, Tongji University, Shanghai, 200092, China (shan_haiying@tongji.edu.cn). Partially supported by NSFC project no. 11271288.

${ }^{\ddagger}$ College of Science, University of Shanghai for Science and Technology, Shanghai, 200093, China (changxiang-he@163.com, zhsh-yu@163.com). 
THEOREM 1.1. Let $e$ be an edge between the $t_{i}$-part and $t_{j}$-part of $K_{t_{1}, \ldots, t_{k}}$. Then

(1) For $k \geq 4$, if $t_{i}=t_{j}=1$, then $\mathcal{E}\left(K_{t_{1}, \ldots, t_{k}}-e\right)<\mathcal{E}\left(K_{t_{1}, \ldots, t_{k}}\right)$, otherwise, $\mathcal{E}\left(K_{t_{1}, \ldots, t_{k}}-e\right)>\mathcal{E}\left(K_{t_{1}, \ldots, t_{k}}\right)$. (2) For $k=3$, if $t_{i}+t_{j} \leq 3$, then $\mathcal{E}\left(K_{t_{1}, \ldots, t_{k}}-e\right)<\mathcal{E}\left(K_{t_{1}, \ldots, t_{k}}\right)$, otherwise, $\mathcal{E}\left(K_{t_{1}, \ldots, t_{k}}-e\right)>\mathcal{E}\left(K_{t_{1}, \ldots, t_{k}}\right)$. (3) For $k=2$, if $\min \left\{t_{i}, t_{j}\right\}=1$, then $\mathcal{E}\left(K_{t_{1}, \ldots, t_{k}}-e\right)<\mathcal{E}\left(K_{t_{1}, \ldots, t_{k}}\right)$, otherwise, $\mathcal{E}\left(K_{t_{1}, \ldots, t_{k}}-e\right)>$ $\mathcal{E}\left(K_{t_{1}, \ldots, t_{k}}\right)$.

This paper is organized as follows. In Section 2, we will give a generalization of Theorem 4 in [1] and some results which will be needed in the next two sections. In the third section, we will determine how the energy of a complete multipartite graph, with at least four parts, changes when an edge is removed. In the last section, we will characterize how the energy of a complete tripartite graph changes when an edge is deleted.

2. Preliminaries. We begin this section with the Interlacing Theorem. By Perron-Frobenius theory, the largest eigenvalue of a connected graph goes down when one removes an edge or a vertex. Interlacing also gives more information about what happens with the $i$-th largest eigenvalues.

LEMma 2.1. (Interlacing) If $G$ is a graph on $n$ vertices with eigenvalues $\lambda_{1}(G) \geq \cdots \geq \lambda_{n}(G)$ and $H$ is an induced subgraph on $m$ vertices with eigenvalues $\lambda_{1}(H) \geq \cdots \geq \lambda_{m}(H)$, then for $i=1, \ldots, m$,

$$
\lambda_{i}(G) \geq \lambda_{i}(H) \geq \lambda_{n-m+i}(G) .
$$

In the next two sections, we will use $\lambda_{2}(G) \geq \lambda_{2}(H)$ (where $H$ is an induced subgraph of $G$ ) many times.

As known, equitable partition represents a powerful tool in spectral graph theory. In this paper, we also use this powerful tool to simplify our calculation.

Given a graph $G$, the partition $V(G)=V_{1} \cup \dot{\cup} V_{2} \dot{\cup} \cdots \dot{\cup} V_{k}$ is an equitable partition if every vertex in $V_{i}$ has the same number of neighbours in $V_{j}$, for all $i, j \in\{1,2, \ldots, k\}$. Suppose $\Pi$ is an equitable partition $V(G)=V_{1} \dot{\cup} V_{2} \dot{\cup} \ldots \dot{\cup} V_{k}$ and that each vertex in $V_{i}$ has $b_{i j}$ neighbours in $V_{j}(i, j \in\{1,2, \ldots, k\})$. The matrix $\left(b_{i j}\right)$ is called the quotient matrix of $\Pi$, denoted by $B_{\Pi}$. The largest eigenvalue of $B_{\Pi}$ is also the spectral radius of $G$ (see [2], Corollary 3.9). In order to determine the spectral radius of graph $G$, we can calculate the largest root of the characteristic polynomial of one of its quotient matrices, which has a lower degree.

For convenience, in this paper, we use $\lambda(G)$ and $\mathbf{x}$, respectively, to denote the spectral radius and the corresponding unit eigenvector of the adjacency matrix of $G$. Suppose $V_{i}$ is the $t_{i}$-part of $K_{t_{1}, t_{2} \ldots, t_{k}}$, then $V_{1} \cup V_{2} \cup \cdots \cup V_{k}$ is an equitable partition. Unless otherwise specified, the cells of equitable partition of $K_{t_{1}, t_{2}, \ldots, t_{k}}$ are $V_{1}, V_{2}, \ldots, V_{k}$. Obviously, vertices in the same part $V_{i}$ have equal x-components, denoted by $x_{i}$.

Akbari, Ghorbani and Oboudi (see Theorem 4 in [1]) proved that for any complete multipartite graph $K_{t_{1}, \ldots, t_{k}}$ with $k \geq 2, t_{i} \geq 2$, then $\mathcal{E}\left(K_{t_{1}, \ldots, t_{k}}-e\right)>\mathcal{E}\left(K_{t_{1}, \ldots, t_{k}}\right)$ for any edge $e$. Using the idea of Akbari, Ghorbani and Oboudi, we get a generalization of this result.

THEOREM 2.2. Let $S$ be a non-empty edge subset of the complete multipartite graph $G=K_{t_{1}, \ldots, t_{k}}$ and $H$ be the corresponding subgraph induced by $S$. Let $V_{i}$ be the $i$-th part of $G$, and $U_{i}=V(H) \cap V_{i}(1 \leq i \leq k)$. If $\left|V_{i}\right| \geq 2 \lambda(H)\left|U_{i}\right|$ holds for any $i$, then we have $\mathcal{E}(G-S)>\mathcal{E}(G)$. 
Proof. Let $A$ and $B$ be the adjacency matrices of $G$ and $G-S$, respectively. We may assume that $B=A-C$, where $C$ is the adjacency matrix of the spanning subgraph of $G$ with only edges in $S$. Let $\mathbf{x}$ be the Perron vector of $A$.

Since each part of $G$ is a cell of an equitable partition of $G$, the vertices of each part have the same corresponding entries in $\mathbf{x}$. By the Rayleigh-Ritz theorem and $\left|V_{i}\right| \geq 2 \lambda(H)\left|U_{i}\right|$ for $1 \leq i \leq k$, we have

$$
\mathbf{x}^{T} C \mathbf{x}=\mathbf{y}^{T} A(H) \mathbf{y} \leq \lambda(H) \mathbf{y}^{T} \mathbf{y} \leq \frac{1}{2} \mathbf{x}^{T} \mathbf{x}=\frac{1}{2}
$$

where $\mathbf{y}$ is the subvector of $\mathbf{x}$ indexed by vertices in $H$.

Thus,

$$
\lambda(B) \geq \mathbf{x}^{T} B \mathbf{x}=\mathbf{x}^{T} A \mathbf{x}-\mathbf{x}^{T} C \mathbf{x} \geq \lambda(A)-\frac{1}{2}
$$

Suppose $e=(u, v) \in S$ and $u \in U_{i}, v \in U_{j}$. Since $S$ is nonempty, $\lambda(H) \geq 1$. So $\left|V_{i}\right| \geq 2\left|U_{i}\right|>\left|U_{i}\right|,\left|V_{j}\right| \geq$ $2\left|U_{j}\right|>\left|U_{j}\right|$. Let $u^{\prime} \in V_{i}-U_{i}$ and $v^{\prime} \in V_{j}-U_{j}$. Then $P_{4}=u v^{\prime} u^{\prime} v$ is an induced subgraph of $G e$. Therefore, by the Interlacing theorem,

$$
\lambda_{2}(B) \geq \lambda_{2}\left(P_{4}\right) \approx 0.618
$$

Thus,

$$
\mathcal{E}(G-S) \geq 2\left(\lambda(B)+\lambda_{2}(B)\right)>2 \lambda(A)=\mathcal{E}(G)
$$

Obviously, Theorem 2.2 generalizes Theorem 4 in [1]. Observe that if $U_{i}=\emptyset$, the condition $\left|V_{i}\right| \geq$ $2 \lambda(H)\left|U_{i}\right|=0$ holds trivially whether $\left|V_{i}\right|$ is 1 or not, so the complete multipartite graph in the above theorem needs not be 1-part free.

Theorem 2.2 immediately implies that deleting any edge between non-1-parts of the complete multipartite graph will increase the energy.

However, deleting one edge between two 1-parts of complete multipartite graph will decrease the energy. If $K_{t_{1}, \ldots, t_{k}}$ has two 1-parts, without loss of generality, we assume $t_{1}=t_{2}=1$, and $e$ is the edge between these 1-parts, then

$$
\mathcal{E}\left(K_{1,1, t_{3}, \ldots, t_{k}}-e\right)=\mathcal{E}\left(K_{2, t_{3}, \ldots, t_{k}}\right)=2 \lambda\left(K_{2, t_{3}, \ldots, t_{k}}\right)<2 \lambda\left(K_{1,1, t_{3}, \ldots, t_{k}}\right)=\mathcal{E}\left(K_{1,1, t_{3}, \ldots, t_{k}}\right) .
$$

In order to completely determine how the energy of complete multipartite graph changes when one edge is removed, we only need to consider the deleted edge is between a 1-part and a non-1-part. So in the next we assume that the considered complete multipartite graph is $K_{1, i, t_{3}, \ldots, t_{k}}$ (where $i \geq 2$ ) and the deleted edge is between 1-part and $i$-part.

Without loss of generality, we assume that $\mathbf{x}$-components of the ends of the deleted edge are $x_{1}$ and $x_{2}$, respectively.

The following lemma is a starting point of our discussions.

LEMMA 2.3. Let $\mathbf{x}$ be a perron vector of complete multipartite graph $G$. Let $e=$ uv be an edge of $G$ and the corresponding entries in $\mathbf{x}$ be $x_{1}$ and $x_{2}$, respectively. If there exists some constant a such that $\lambda_{2}(G-e)>a$ and $x_{1}^{2}+x_{2}^{2} \leq a$, then $\mathcal{E}(G-e)>\mathcal{E}(G)$. 
Proof. Let $A(G-e)=A(G)-C$, where $C$ is the adjacency matrix of the spanning subgraph of $G$ with only one edge $e$. Then

$$
\mathbf{x}^{T} C \mathbf{x}=2 x_{1} x_{2} \leq x_{1}^{2}+x_{2}^{2} \leq a .
$$

By the Rayleigh-Ritz theorem,

$$
\lambda(G-e) \geq \mathbf{x}^{T} A(G-e) \mathbf{x}=\mathbf{x}^{T} A(G) \mathbf{x}-\mathbf{x}^{T} C \mathbf{x} \geq \lambda(G)-a .
$$

Because $\lambda_{2}(G-e)>a$, we arrive at

$$
\mathcal{E}(G-e) \geq 2\left(\lambda(G-e)+\lambda_{2}(G-e)\right)>2 \lambda(G)=\mathcal{E}(G) .
$$

Next we will give a lower bound on the spectral radius of complete multipartite graph $K_{1, i, t_{3}, \ldots, t_{k}}$ which will be used in the calculation in the subsequent sections.

Lemma 2.4. Let $G=K_{1, i, t_{3}, \ldots, t_{k}}$ be a complete multipartite graph with order $n$. We have:

(1) If $k \geq 3$, then $\lambda(G)>\sqrt{(n-i)(i+1)}$.

(2) In particular, if $2 \leq i \leq n-5$ and $\max \left\{t_{3}, \ldots, t_{k}\right\}=1$, then $\lambda(G)>n-i+0.67$ holds.

Proof. (1) The characteristic polynomial of the quotient matrix of $K_{1, i, n-i-1}$ is

$$
f(x)=x^{3}-((n-i)(i+1)-1) x-2(n-i-1) i .
$$

It is easy to see that $\lambda\left(K_{1, i, n-i-1}\right)>\sqrt{(n-i)(i+1)}$. Note that $K_{1, i, n-i-1}$ is a subgraph of $G$, so that, $\lambda(G) \geq \lambda\left(K_{1, i, n-i-1}\right)>\sqrt{(n-i)(i+1)}$.

(2) If $\max \left\{t_{3}, \ldots, t_{k}\right\}=1$, then

$$
Q=\left(\begin{array}{rr}
0 & n-i \\
i & n-i-1
\end{array}\right)
$$

is a quotient matrix of $G$, so $\lambda(G)$ is the largest root of $\phi(Q, x)=x^{2}-(n-i-1) x-i(n-i)$.

If $2 \leq i \leq n-5$, then we have

$$
\begin{aligned}
\phi(Q, n-i+0.67) & =i^{2}-(i-1.67) n-1.67 i+1.1189 \\
& \leq i^{2}-(i-1.67)(i+5)-1.67 i+1.1189 \\
& =9.4689-5 i<0
\end{aligned}
$$

Therefore, $\lambda(G)>n-i+0.67$.

The following lemma provides some sufficient (but not necessary) conditions for $\mathcal{E}(G-e)>\mathcal{E}(G)$, and is also a key tool which will be widely used in the sequel proofs.

LEMMA 2.5. Let $G=K_{1, i, t_{3}, \ldots, t_{k}}$ be a complete $k$-partite graph ( $\left.k \geq 3\right)$ with order $n$, and $e$ be an edge between 1-part and $i$-part. Suppose that $a$ is a positive constant and $\lambda_{2}(G-e)>a$. If one of the following holds:

(1) $\frac{2 i+1}{i(i+2)}<a<1$ and $f_{a}(n, i)=n\left(a i^{2}-2(1-a) i-1\right)-a i^{3}+(1-a) i^{2}-(a-2) i>0$,

(2) $\frac{2(n-1)}{\lambda^{2}+n-1}<a$, where $\lambda=\lambda(G)$,

then $\mathcal{E}(G-e)>\mathcal{E}(G)$. 
Proof. By Lemma 2.3, it suffices to prove $x_{1}^{2}+x_{2}^{2} \leq a$.

(1) Let $a>\frac{2 i+1}{i(i+2)}$, which means $a i^{2}-2(1-a) i-1>0$. Combining this with $f_{a}(n, i)>0$, i.e., $n \geq \frac{a i^{3}-(1-a) i^{2}+(a-2) i}{a i^{2}-2(1-a) i-1}$, we can get $\lambda^{2}(G)>(n-i)(i+1) \geq \frac{(n-1)(1-a) i}{a i-1}$, which yields that

$$
\frac{(n-1) i}{\lambda^{2}(G)}<\frac{a i-1}{1-a}
$$

From the eigenvalue equation of $G$, we have $\lambda(G) x_{1}=i x_{2}+t_{3} x_{3}+\cdots+t_{k} x_{k}$. Applying the Cauchy-Schwarz inequality, we see that

$$
\lambda^{2}(G) x_{1}^{2} \leq\left(i+t_{3}+\cdots+t_{k}\right)\left(i x_{2}^{2}+t_{3} x_{3}^{2}+\cdots+t_{k} x_{k}^{2}\right)=(n-1)\left(i x_{2}^{2}+m\right)=(n-1)\left(1-x_{1}^{2}\right),
$$

where $m=t_{3} x_{3}^{2}+\cdots+t_{k} x_{k}^{2}$. Hence, $x_{1}^{2} \leq \frac{(n-1)}{\lambda^{2}(G)}\left(i x_{2}^{2}+m\right) \leq \frac{a i-1}{1-a} x_{2}^{2}+\frac{a}{1-a} m$. This shows that

$$
x_{1}^{2}+x_{2}^{2} \leq \frac{a}{1-a}\left((i-1) x_{2}^{2}+m\right)=\frac{a}{1-a}\left(1-x_{1}^{2}-x_{2}^{2}\right) \text {, }
$$

which implies that $x_{1}^{2}+x_{2}^{2} \leq a$ holds.

(2) By considering eigenvalue equations $\lambda x_{1}=i x_{2}+t_{3} x_{3}+\cdots+t_{k} x_{k}$ and $\lambda x_{2}=x_{1}+t_{3} x_{3}+\cdots+t_{k} x_{k}$, we find $x_{2}=\left(\frac{\lambda+1}{\lambda+i}\right) x_{1}$. From $\lambda^{2} x_{1}^{2} \leq(n-1)\left(1-x_{1}^{2}\right)$, we have

$$
x_{1}^{2} \leq \frac{n-1}{\lambda^{2}+n-1} .
$$

Therefore,

$$
x_{1}^{2}+x_{2}^{2}=\left(1+\left(\frac{\lambda+1}{\lambda+i}\right)^{2}\right) x_{1}^{2} \leq\left(1+\left(\frac{\lambda+1}{\lambda+i}\right)^{2}\right) \frac{n-1}{\lambda^{2}+n-1}<\frac{2(n-1)}{\lambda^{2}+n-1}<a .
$$

3. The complete multipartite graph with at least four parts. In this section, we consider how

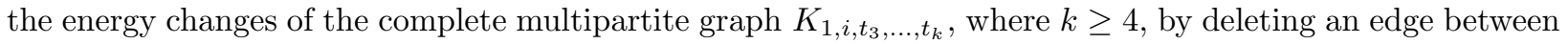
1 -part and $i$-part. We will distinguish into two cases $i \geq 4$ and $i \in\{2,3\}$, and will apply the two methods in Lemma 2.5 to compare the energies of $K_{1, i, t_{3}, \ldots, t_{k}}-e$ and $K_{1, i, t_{3}, \ldots, t_{k}}$. Now we consider the case of $i \geq 4$ firstly.

LEMMA 3.1. If $k \geq 4$ and $i \geq 4$, then $\mathcal{E}\left(K_{1, i, t_{3}, \ldots, t_{k}}-e\right)>\mathcal{E}\left(K_{1, i, t_{3}, \ldots, t_{k}}\right)$ for any edge e between 1-part and $i$-part.

Proof. As $k \geq 4, K_{1,4,1,1}-e$ is an induced subgraph of $K_{1, i, t_{3}, \ldots, t_{k}}-e$, by the Interlacing Theorem $\lambda_{2}\left(K_{1, i, t_{3}, \ldots, t_{k}}-e\right) \geq \lambda_{2}\left(K_{1,4,1,1}-e\right)=\sqrt{2}-1>0.4$ holds.

Since $\frac{2 i+1}{i(i+2)}$ is a decreasing function for $i$, we have $\frac{2 i+1}{i(i+2)} \leq \frac{3}{8}<0.4$ for $i \geq 4$. Now we use Lemma 2.5 by taking $a=0.4$, then

$$
5 f_{0.4}(n, i) \geq 5 f_{0.4}(i+3, i)=3\left(i^{2}-5 i-5\right)>0
$$

holds for all $i \geq 6$. Hence, $\mathcal{E}\left(K_{1, i, t_{3}, \ldots, t_{k}}-e\right)>\mathcal{E}\left(K_{1, i, t_{3}, \ldots, t_{k}}\right)$ holds when $i \geq 6$.

Because $f_{0.414}(n, 4)>0$ when $n \geq 12$, and $f_{0.414}(n, 5)>0$ when $n \geq 9$, and these show that $\mathcal{E}\left(K_{1, i, t_{3}, \ldots, t_{k}}-e\right)>\mathcal{E}\left(K_{1, i, t_{3}, \ldots, t_{k}}\right)$ holds for $i=4,5$ when $n \geq 12$ and $n \geq 9$, respectively. With the aid of mathematics software "SageMath" [6], one can verify the result holds for $i=4, n \leq 11$ and $i=5, n \leq 8$. 
The next lemma offers a method to compare the spectral radius of two complete multipartite graphs with the same order, which will be used in the proof of Lemma 3.3.

LEMMA 3.2. ([7]) If $n_{i}-n_{j} \geq 2$, then $\lambda\left(K_{n_{1}, \ldots, n_{i}-1, \ldots, n_{j}+1, \ldots, n_{p}}\right)>\lambda\left(K_{n_{1}, \ldots, n_{i}, \ldots, n_{j}, \ldots, n_{p}}\right)$.

LEMma 3.3. $\mathcal{E}\left(K_{1, i, t_{3}, \ldots, t_{k}}-e\right)>\mathcal{E}\left(K_{1, i, t_{3}, \ldots, t_{k}}\right)$ for any $k \geq 4, i \in\{2,3\}$ and every edge e between 1-part and $i$-part.

Proof. For short, we write $K_{1, i, t_{3}, \ldots, t_{k}}$ as $G$.

By Lemma 2.4, when $n \geq 8$, if $\max \left\{t_{3}, \ldots, t_{k}\right\}=1, \lambda(G)>n-2.33$ holds, which implies that $\frac{2(n-1)}{\lambda^{2}+n-1}<$ $\frac{2(n-1)}{(n-2.33)^{2}+n-1}$. Note that $\frac{2(n-1)}{(n-2.33)^{2}+n-1}<0.357$ when $n \geq 8$. Hence, $\frac{2(n-1)}{\lambda^{2}+n-1}<0.357$

If $\max \left\{t_{3}, \ldots, t_{k}\right\} \geq 2$, say $t_{3} \geq 2$, then $K_{1, i, t_{3}, n-i-t_{3}-1}$ is a subgraph of $G$, so

$$
\lambda(G) \geq \lambda\left(K_{1, i, t_{3}, n-i-t_{3}-1}\right) \geq \lambda\left(K_{1, i, 2, n-i-3}\right) \geq \lambda\left(K_{1,2,2, n-5}\right),
$$

the last two inequalities follow from Lemma 3.2. Note that $\lambda\left(K_{1,2,2, n-5}\right)$ is the largest root of $g(x)=x^{4}-$ $(5 n-17) x^{2}-8(2 n-9) x-6(2 n-10)$ which is the characteristic polynomial of its equitable matrix. It is easy to check that $\tau(g)>\sqrt{5 n-7}$ when $n \geq 8$, which means $\lambda(G)>\sqrt{5 n-7}$. And thus, $\frac{2(n-1)}{\lambda^{2}+n-1}<\frac{2(n-1)}{6 n-8}<0.357$, when $n \geq 8$.

On the other hand, $\lambda_{2}(G-e) \geq \lambda_{2}\left(K_{1,2,1,1}-e\right)>0.357$. Now we use (2) of Lemma 2.5 by taking $a=0.357$, we have $\mathcal{E}(G-e)>\mathcal{E}(G)$.

With the aid of mathematics software "SageMath" [6], one can verify the result holds for $G$ when $n \leq 7$.

The following proposition is an immediate result from Lemma 3.1 and Lemma 3.3.

Proposition 3.4. $\mathcal{E}\left(K_{1, i, t_{3}, \ldots, t_{k}}-e\right)>\mathcal{E}\left(K_{1, i, t_{3}, \ldots, t_{k}}\right)$ holds for any $k \geq 4, i \geq 2$ and e between 1-part and $i$-part.

4. Complete tripartite graph. In this section, we will focus on the energy change of the complete tripartite graph $K_{1, i, n-i-1}$. We distinguish into two cases: $4 \leq i \leq n-3$ and $i \in\{2,3, n-2\}$. The proof of the first case is similar to the proof of Lemma 3.1. But for the case $i \in\{2,3, n-2\}$, it is almost impossible to use the former method, so we will give another new energy comparison method.

LEMma 4.1. If $4 \leq i \leq n-3$, then $\mathcal{E}\left(K_{1, i, n-i-1}-e\right)>\mathcal{E}\left(K_{1, i, n-i-1}\right)$, for any edge e between 1-part and $i$-part.

Proof. If $i \geq 8$, with the similar manner of Lemma 3.1, $K_{1,5,2}-e$ is an induced subgraph of $K_{1, i, n-i-1}-e$, by the Interlacing Theorem, $\lambda_{2}\left(K_{1, i, n-i-1}-e\right) \geq \lambda_{2}\left(K_{1,5,2}-e\right)>\frac{11}{30}$. that

Taking $a=\frac{11}{30}$, we find $f_{a}(n, i)$ is a strictly increasing function for $n$. Since $n \geq i+3$, we easily have

$$
30 f_{a}(n, i) \geq 30 f_{a}(i+3, i)=14 i^{2}-95 i-90>0
$$

holds when $i \geq 8$. Since $\frac{2 i+1}{i(i+2)} \leq \frac{17}{80}<a$ for $i \geq 8$, by (1) of Lemma 2.5, we have $\mathcal{E}\left(K_{1, i, n-i-1}-e\right)>$ $\mathcal{E}\left(K_{1, i, n-i-1}\right)$. 
When $4 \leq i \leq 7$, we take $a=0.36<\lambda_{2}\left(K_{1,4,2}-e\right)$. Lemma 2.4 provides that $\frac{2(n-1)}{\lambda^{2}+n-1}<\frac{2(n-1)}{(n-i)(i+1)+n-1}<$ $\lambda_{2}\left(K_{1,4,2}-e\right)$ for $n \geq 35$. Since $\lambda_{2}\left(K_{1, i, n-i-1}-e\right)>\lambda_{2}\left(K_{1,4,2}-e\right), \mathcal{E}\left(K_{1, i, n-i-1}-e\right)>\mathcal{E}\left(K_{1, i, n-i-1}\right)$ follows from (2) of Lemma 2.5 .

With the aid of mathematics software "SageMath" [6], one can verify the result holds for $n \leq 34$.

Next we will consider how the energy changes of $K_{1, n-2,1}, K_{1,2, n-3}$ and $K_{1,3, n-4}$ by deleting one edge between the first two parts. For convenience, we use $\tau(f)$ to denote the largest real root of the equation $f(x)=0$ if it exists.

The following is a lemma about the largest root of equation which will be used in the proof of our last lemma.

LEMmA 4.2. Let $f(x)=x^{4}+a x^{2}+b x+c$ and $g(x)=x^{6}+8 a x^{4}+16\left(a^{2}-4 c\right) x^{2}-64 b^{2}$. If all roots of the equation $f(x)=0$ are real, then:

(1) $g(x)=0$ has only real roots.

(2) In particular, if $f(x)=0$ has exactly two positive roots, then $\mathcal{E}(f)=\tau(g)$.

Proof. Let $x_{1}, x_{2}, x_{3}, x_{4}$ be the four real roots of $f(x)=0$, then

$$
\begin{aligned}
x_{1}+x_{2}+x_{3}+x_{4} & =0, \\
x_{1} x_{2}+x_{1} x_{3}+x_{2} x_{3}+x_{1} x_{4}+x_{2} x_{4}+x_{3} x_{4} & =a, \\
x_{1} x_{2} x_{3}+x_{1} x_{2} x_{4}+x_{1} x_{3} x_{4}+x_{2} x_{3} x_{4} & =-b, \\
x_{1} x_{2} x_{3} x_{4} & =c .
\end{aligned}
$$

(1) Put $y=2\left(x_{1}+x_{2}\right)$. By formulas (4.1) and (4.2), we see that

$$
x_{1} x_{2}+x_{3} x_{4}=a+\left(x_{1}+x_{2}\right)^{2}=a+\frac{y^{2}}{4} .
$$

On the other hand,

$$
y\left(x_{1} x_{2}-x_{3} x_{4}\right)=2 b
$$

follows from formulas (4.1) and (4.3). Then $\left(a+\frac{y^{2}}{4}\right)^{2} y^{2}-4 b^{2}=4 x_{1} x_{2} x_{3} x_{4} y^{2}=4 c y^{2}$, which yields

$$
y^{6}+8 a y^{4}+16\left(a^{2}-4 c\right) y^{2}-64 b^{2}=0 .
$$

That is to say, $2\left(x_{1}+x_{2}\right)$ is a root of $g(x)=0$. From the symmetry of $x_{1}, x_{2}, x_{3}, x_{4}$, we know that $2\left(x_{1}+x_{3}\right), 2\left(x_{1}+x_{4}\right), 2\left(x_{2}+x_{3}\right), 2\left(x_{2}+x_{4}\right), 2\left(x_{3}+x_{4}\right)$ are roots of $g(x)=0$. In view of $g(x)=0$ has exactly 6 roots, then all roots of $g(x)=0$ are $2\left(x_{1}+x_{2}\right), 2\left(x_{1}+x_{3}\right), 2\left(x_{1}+x_{4}\right), 2\left(x_{2}+x_{3}\right), 2\left(x_{2}+x_{4}\right), 2\left(x_{3}+x_{4}\right)$ which are all real.

(2) If $x_{1}, x_{2}$ are positive, and $x_{3}, x_{4}$ are negative, then $\tau(g)=2\left(x_{1}+x_{2}\right)$. Note that $\mathcal{E}(f)=x_{1}+x_{2}-$ $x_{3}-x_{4}=2\left(x_{1}+x_{2}\right)$ implies that $\tau(g)=\mathcal{E}(f)$.

Now we are ready to determine how the energy changes of $K_{1, i, n-i-1}$ due to deleting one edge between 1 -part and $i$-part, where $i \in\{2,3, n-2\}$. 
Lemma 4.3. If $e$ is an edge between 1-part and $i$-part in $K_{1, i, n-i-1}, i \in\{2,3, n-2\}$. Then

(1) $\mathcal{E}\left(K_{1,2, n-3}-e\right)<\mathcal{E}\left(K_{1,2, n-3}\right)$,

(2) $\mathcal{E}\left(K_{1,3, n-4}-e\right)>\mathcal{E}\left(K_{1,3, n-4}\right)$,

(3) $\mathcal{E}\left(K_{1, n-2,1}-e\right)>\mathcal{E}\left(K_{1, n-2,1}\right)$.

Proof. For short, we write $K_{1, i, n-i-1}$ as $G$, and $n-i-1$ as $t$, where $i \in\{2,3, n-2\}$. Then

$$
Q=\left(\begin{array}{ccc}
0 & i & t \\
1 & 0 & t \\
1 & i & 0
\end{array}\right),
$$

is a quotient matrix of $G$, and

$$
\Phi(Q, x)=x^{3}-(t i+i+t) x-2 t i .
$$

It is easy to see that $\Phi(Q, x)=0$ has two negative roots, say $-x_{1},-x_{2}$, and one positive root, say $x_{3}$. Then we have $-x_{1}-x_{2}+x_{3}=0$ and $\mathcal{E}(G)=\mathcal{E}(Q)=2 x_{3}$. If we denote $g(x)=8 \phi\left(Q, \frac{x}{2}\right)=x^{3}-4(t i+i+t) x-16 t i$, then $\mathcal{E}(G)=\tau(g)$.

Similarly,

$$
Q^{\prime}=\left(\begin{array}{rrrr}
0 & 0 & 0 & t \\
0 & 0 & i-1 & t \\
0 & 1 & 0 & t \\
1 & 1 & i-1 & 0
\end{array}\right)
$$

is a quotient matrix of $G-e$, and

$$
\Phi\left(Q^{\prime}, x\right)=x^{4}+(-t i-i-t+1) x^{2}-2(t i-t) x+t i-t .
$$

Obviously, $\Phi\left(Q^{\prime}, x\right)=0$ has exactly two positive roots. Applying Lemma 4.2 to $\Phi\left(Q^{\prime}, x\right)$, we can obtain $h(x)=x^{6}-8(t i+t+i-1) x^{4}+16\left((t i+t)^{2}+(i-1)^{2}(2 t+1)\right) x^{2}-256(t i-t)^{2}$, such that $\mathcal{E}(G-e)=\mathcal{E}\left(Q^{\prime}\right)=\tau(h)$.

Let

$$
\begin{aligned}
& q(x)=x^{3}-4((i+1) t+i-2) x+16 t i, \\
& r(x)=h(x)-q(x) g(x)=-16\left[(4 t i-4 t-1) x^{2}-8 t i x-16(2 i-1) t^{2}\right] .
\end{aligned}
$$

Since $q(x)-g(x)=8 x+32, \tau(q)<\tau(g)$ and $\tau(g)=\tau(q g)$.

Case 1. Suppose $i=2$. Then

$$
\begin{aligned}
& h(x)=\left(x^{3}+4 x^{2}-(12 t-4) x-16 t\right)\left(x^{3}-4 x^{2}-(12 t-4) x+16 t\right), \\
& g(x)=x^{3}-4(2+3 t) x-32 t .
\end{aligned}
$$

Suppose

$$
\begin{aligned}
& h_{1}(x)=x^{3}+4 x^{2}-(12 t-4) x-16 t, \quad h_{2}(x)=x^{3}-4 x^{2}-(12 t-4) x+16 t, \\
& r_{2}(x)=\frac{1}{4}\left(h_{2}(x)-g(x)\right)=-x^{2}+3 x+12 t .
\end{aligned}
$$

Then $h_{1}(x)-g(x)>0$ when $x>0$, so $\tau\left(h_{1}\right)<\tau(g)$. Since $h_{2}(x)=(1-x) r_{2}(x)+x+4 t, h_{2}(x)>0$ always holds for any $x>\tau\left(r_{2}\right)>1$. This means all the positive roots of $h_{2}(x)$ are in the interval $\left(0, \tau\left(r_{2}\right)\right)$. On the 
other hand, we find that $h_{2}(x)>g(x)$ holds in $\left(0, \tau\left(r_{2}\right)\right)$. Combining these with the fact $g(x)=0$ has exactly one positive root, we conclude $\tau(g)>\tau\left(h_{2}\right)$. Consequently, $\mathcal{E}(G)>\max \left\{\tau\left(h_{1}\right), \tau\left(h_{2}\right)\right\}=\tau(h)=\mathcal{E}(G-e)$, Hence, (1) holds.

Case 2. Suppose $i=3$. Note that $g(2 \sqrt{4 t+3})=-48 t<0$, and thus, $\tau(g)>2 \sqrt{4 t+3}$. Since $r(x)=-16\left((8 t-1) x^{2}-24 t x-80 t^{2}\right)$ has only one positive root, say $x_{0}$, so $r(x)$ is a decreasing function for $x>x_{0}$. It is easy to see $x_{0}<2 \sqrt{4 t+3}$ when $t \geq 2$. Therefore, $h(\tau(g))=r(\tau(g))<r(2 \sqrt{4 t+3})<0$ for $t \geq 2$. Hence, $h(\tau(g))=r(\tau(g))<0$, and then $\tau(h)>\tau(g)$, i.e., $\mathcal{E}(G-e)>\mathcal{E}(G)$.

Case 3. Suppose $i=n-2$. Then $t=1$, and $g(x)=\left(x^{2}-2 x-8 i\right)(x+2)$, which yields that $\mathcal{E}(G)=\tau(g)=1+\sqrt{1+8 i}$.

Note that $h(1+\sqrt{1+8 i})=r(1+\sqrt{1+8 i})=32\left(-16 i^{2}+36 i+5 \sqrt{8 i+1}-3\right)$ is a decreasing function for $i \geq 3$. Hence, $h(1+\sqrt{1+8 i}) \leq h(6)=-448<0$ when $i \geq 3$. Consequently, $\mathcal{E}(G-e)=\tau(h)>$ $1+\sqrt{1+8 i}=\mathcal{E}(G)$.

The following proposition is an immediate result from Lemma 4.1 and Lemma 4.3.

Proposition 4.4.

(1) $\mathcal{E}\left(K_{1,2, n-3}-e\right)<\mathcal{E}\left(K_{1,2, n-3}\right)$ for any edge e between 1-part and 2-part.

(2) $\mathcal{E}\left(K_{1, i, n-i-1}-e\right)>\mathcal{E}\left(K_{1, i, n-i-1}\right)$ for $i \geq 3$ and any edge e between 1 -part and $i$-part.

Combining these with the well-known results of bipartite graphs, we can get our main result.

THEOREM 4.5. Let $e$ be an edge between the $t_{i}$-part and $t_{j}$-part of $K_{t_{1}, \ldots, t_{k}}$. Then:

(1) For $k \geq 4$, if $t_{i}=t_{j}=1$, then $\mathcal{E}\left(K_{t_{1}, \ldots, t_{k}}-e\right)<\mathcal{E}\left(K_{t_{1}, \ldots, t_{k}}\right)$, otherwise, $\mathcal{E}\left(K_{t_{1}, \ldots, t_{k}}-e\right)>\mathcal{E}\left(K_{t_{1}, \ldots, t_{k}}\right)$.

(2) For $k=3$, if $t_{i}+t_{j} \leq 3$, then $\mathcal{E}\left(K_{t_{1}, \ldots, t_{k}}-e\right)<\mathcal{E}\left(K_{t_{1}, \ldots, t_{k}}\right)$, otherwise, $\mathcal{E}\left(K_{t_{1}, \ldots, t_{k}}-e\right)>\mathcal{E}\left(K_{t_{1}, \ldots, t_{k}}\right)$.

(3) For $k=2$, if $\min \left\{t_{i}, t_{j}\right\}=1$, then $\mathcal{E}\left(K_{t_{1}, \ldots, t_{k}}-e\right)<\mathcal{E}\left(K_{t_{1}, \ldots, t_{k}}\right)$, otherwise, $\mathcal{E}\left(K_{t_{1}, \ldots, t_{k}}-e\right)>$ $\mathcal{E}\left(K_{t_{1}, \ldots, t_{k}}\right)$.

\section{REFERENCES}

[1] S. Akbari, E. Ghorbani, and M. Oboudi. Edge addition, singular values, and energy of graphs and matrices. Linear Algebra Appl., 430:2192-2199, 2009.

[2] D. Cvetković, P. Rowlinson, and S. Simic. An Introduction to the Theory of Graph Spectra. Cambridge University Press, Cambridge, 2010.

[3] J. Day and W. So. Graph energy change due to edge deletion. Linear Algebra Appl., 428:2070-2078, 2008.

[4] I. Gutman. The energy of a graph: Old and new results. In: A. Betten, A. Kohnert, R. Laue, and A. Wassermann (editors), Algebraic Combinatorics and Applications, Springer-Verlag, Berlin, 196-211, 2001.

[5] I. Gutman. The energy of a graph. Ber. Math-Statist. Sekt. Forschungszentrum Graz, 103:1-22, 1978.

[6] W.A. Stein, et al. Sage Mathematics Software (Version 7.3). The Sage Development Team, http:// www.sagemath.org, 2016.

[7] D. Stevanović, I. Gutman, and M.U. Rehman. On spectral radius and energy of complete multipartite graphs. Ars Math. Contemp., 9:109-113, 2015. 